\title{
The leading Ruelle resonances of chaotic maps
}

\author{
Galya Blum ${ }^{1}$ and Oded Agam ${ }^{2}$ \\ 1 Department of Condensed Matter Physics, Weizmann Institute of Science, 76100 Rehovot, Israel \\ 2 The Racah Institute of Physics, The Hebrew University, Jerusalem 91904 Israel
}

\begin{abstract}
The leading Ruelle resonances of typical chaotic maps, the perturbed cat map and the standard map, are calculated by variation. It is found that, excluding the resonance associated with the invariant density, the next subleading resonances are, approximately, the roots of the equation $z^{4}=\gamma$, where $\gamma$ is a positive number which characterizes the amount of stochasticity of the map. The results are verified by numerical computations, and the implications to the form factor of the corresponding quantum maps are discussed.
\end{abstract}

PACS numbers: 05.45.Ac, 05.45.Mt, 03.65.Sq

The statistical charectaristics of the quantum mechanical spectrum of a system, which has a classical analog, are related to its underlying classical dynamics. For example, in chaotic system, this relation is revealed by Gutzwiller's trace formulal which expresses the density of states in terms of a sum over the classical periodic orbits of the system. In disordered systems, spectral properties of the quantum system are expressed in terms of the diffusons and the Cooperons diagrams associated with the classical diffusive modes of the system 2 .

There is, however, a difference between the periodic orbit picture and the diagrammatical approach of disordered systems. The first uses the individual trajectories of the systems as the basic ingredients for the semiclassical expansions, whereas the second approach employs spectral properties of the evolution operator of classical distribution functions. The latter approach is, therefore, more suitable for a field theoretic treatment.

Recently an extension of the field theoretic formalism, from disordered systems to general chaotic ones, has been proposed 3 . The analog of classical diffusion, in the generalized case, is the coarse grained Liouville dynamics of distribution functionst in the limit of zero coarse grainingl. The corresponding evolution operator is the Frobenius-Perron (FP) operator, and its eigenvalues are the Ruelle resonancest. These eigenvalues describe the decay of classical correlation functions of smooth observables.

The association of spectral properties of quantum chaotic systems with their classical spectra calls for a study of the Ruelle resonances of general chaotic systems. The central question is whether chaotic systems can be classified into equivalence classes according to their classical spectrum? An obvious example of such a class is systems where the effective dynamics is diffusive. For instance, the kicked rotor map exhibits diffusion in momentum spaced, and the stadium billiard, in the limit of small distance between the semicircles, is diffusive in the angular momentum space 8 .

Are there other universality classes? In principle, there should be. Diffusion is not the only type of universal dynamics of chaotic systems where correlations are rapidly lost. Other possibilities can be, for instance, various types of Levy flights 2 .

Yet, the lack of approximation schemes for calculating Ruelle resonances, obstructs the characterization of the effective dynamics of general chaotic systems. The physical approach for calculating Ruelle resonances is to project the dynamics, from the full phase space, down to the manifold on which the dynamics is slow, and to construct an equation for the probability density on this manifold. For example, in disordered systems, the fast and the slow manifolds are the momentum and the real space respectively. Projecting the dynamics down to real space yields the diffusion equation, whose eigenvalues constitute the leading Ruelle resonances of the system. In trying to apply a similar procedure for general chaotic systems, one encounters the problem of identifying the slow and the fast manifolds. These can be complicated functions in phase space.

In this paper we choose a different approach. We will construct a simple variational method for calculating the leading resonances, and apply it to two generic maps: The perturbed cat map 10 and the standard mapt. The results indicate that, indeed, the leading Ruelle resonances of these maps share some universal features (different from diffusion). Namely, the configuration of the leading resonances in the complex plain is similar. Excluding the eigenvalue associated with the invariant density, $z_{0}=1$, the next subleading resonances are, approximately, the roots of the equation: $z^{4}=\gamma$, where $\gamma$ is a real positive number, smaller than unity, which decreases as the map becomes more stochastic. It will be shown that this configuration of resonances is significant for the spectral statistics of the corresponding quantum maps. For example, in the form factor, it leads to a suppression of the nonunirersal corrections to the results of random matrix theory 1 (RMT).

In introducing our ideas it will be instructive to consider a mecific example. We begin with the perturbed cat map 10 which is an area preserving map from the unit torus to itself:

$$
\begin{aligned}
& x_{n+1}=2 x_{n}+y_{n} \\
& y_{n+1}=3 x_{n}+2 y_{n}+f\left(x_{n+1}\right) \quad(\operatorname{Mod} 1) .
\end{aligned}
$$

Here $\left(x_{n}, y_{n}\right)$ are the phase space coordinates at discrete 

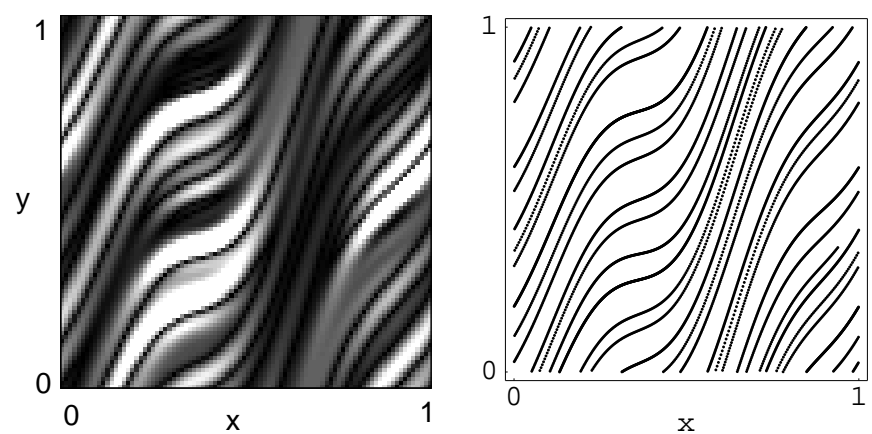

FIG. 1. Left panel: The absolute value of the right eigenfunction, $\psi_{1}^{R}$ of the perturbed cat map at $k=0.5$ and coarse graining $s=0.001$. The dark and the bright regions correspond to the minima and maxima of $\left|\psi_{1}^{R}\right|$, respectively. This function follows the unstable manifold of the map which is shown on the right panel.

time $n$, and

$$
f(x)=\frac{k}{2 \pi}[\cos (2 \pi x)-\cos (4 \pi x)]
$$

is a perturbation function. This function is smooth and periodic on the torus, therefore, the map is continuous, and differentiable. At $k=0$ the map, known as Arnold's cat map 12, is highly chaotid 13 . Namely, time correlation functions decay faster than exponential. This behavior is related to the presence of hidden symmetries of number theoretical origin 14. The role of $f(x)$ is to break these symmetries. As a result, at finite values of $k$, relaxation is exponential. It has been also proved that for $k<0.11$ all the fixed points of (11) are in one to one correspondence with the original cat map. Consequently, all orbits are unstable and the system can be characterized as "hard chaotic" 10. is

The classical propagator of the map, for one time step,

$\left\langle x^{\prime}, y^{\prime}|U| x, y\right\rangle=\delta_{p}\left[x^{\prime}-2 x-y\right] \delta_{p}\left[y^{\prime}-3 x-2 y-f\left(x^{\prime}\right)\right]$,

where $\delta_{p}(x)$ is a periodic $\delta$-function on the torus. Due to Liouville's theorem, the above propagator is unitary when acting on Hilbert space of square integrable functions. Within this space the dynamics is reversible, however, as soon as the dynamics is coarse grained, it becomes irreversible and phase space densities relax to the invariant density.

A simple way of coarse graining the dynamics is to give the $\delta$-functions in (2) a finite width, for instance, by the replacement

$$
\delta_{p}(x) \rightarrow \sum_{n} \frac{1}{\sqrt{\pi s}} e^{-\frac{(x-n)^{2}}{s}}
$$

where $s$ is the coarse graining parameter. The Ruelle resonances of the map are obtained by diagonalizing the propagator at a finite value of $s$, and taking $s$ to zero after the diagonalization. It can be shown that this procedure is equivalent to the introduction of noise into the equations of motion (11), calculation of the eigenvalues averaged oyer the noise, and finally taking the limit of noise to zero 15 . The resulting resonances have absolute values smaller than unity, except for the resonance associates with the invariant density, $z_{0}=1$.

Since the coarse grained evolution operator is not unitary, the left eigenfunctions of $U$ are not the complex conjugates of the right eigenfunctions. Instead they can be viewed as the right eigenfunctions of the propagator associated with the inverse map (i.e. the propagator taking the system one time step backward). Thus the eigenvalue equations are:

$$
U\left|\psi_{\alpha}^{R}\right\rangle=z_{\alpha}\left|\psi_{\alpha}^{R}\right\rangle, \quad\left\langle\psi_{\alpha}^{L}\right| U=\left\langle\psi_{\alpha}^{L}\right| z_{\alpha},
$$

where $\left|\psi_{\alpha}^{R}\right\rangle$ and $\left\langle\psi_{\alpha}^{L}\right|$ are the right and the left eigenfunctions associated with the resonance $z_{\alpha}$. According to Ruelle's theorem6, these eigenfunctions become distributions in the limit of zero coarse graining. The distributions associated with the left and the right eigenfunctions follow the stable and the unstable manifolds respectively. Nevertheless, they satisfy the normalization condition

$$
\left\langle\psi_{\alpha}^{L} \mid \psi_{\beta}^{R}\right\rangle=\delta_{\alpha, \beta} .
$$

In the left panel of Fig. 1 we show an example of the right eigenfunction, $\psi_{1}^{R}$, of the perturbed cat map for $k=0.5$ and $s=0.001$. As can be seen, this eigenfunction follows the unstable manifold depicted on the right panel. As the coarse graining parameter, $s$, reduces, the eigenfunction becomes more singular, in accordance with Ruelle's theorem.

In what follows we shall calculate the leading Ruelle resonances of the map (11) using variational approach which takes into account the structure of the eigenfunctions illustrated in Fig. 1. For this purpose we construct the functional

$$
F=\langle L|U| R\rangle-z\langle L \mid R\rangle,
$$

where $|R\rangle$ and $\langle L|$ are the right and the corresponding left trail functions. The leading Ruelle resonances are obtained by variation 16 of $F$ with respect to $\langle L|$.

The success of a variational calculation depends on the extent to which the variational wave functions capture the physics of the problem. In our case, one should construct left and right trail functions which follow the stable and the unstable manifolds respectively. We will use the dynamics itself to generate these functions by repeated applications of the map on a smooth initial state. Thus, our right trail function takes the form

$$
|R\rangle=\sum_{n=-\infty}^{\infty} A_{n}|n\rangle, \text { with }|n\rangle=e^{2 \pi i \phi_{n}^{R}(x, y)},
$$

where $A_{n}$ are amplitudes to be determined by variation, and the phases $\phi_{n}^{R}(x, y)$ are defined by the recursion equation: 


$$
\phi_{n}^{R}(x, y)=\phi_{n-1}^{R}(2 x-y+f(x), 2 y-3 x-2 f(x))
$$

with the initial phase $\phi_{1}^{R}(x, y)=x$. We define $\phi_{-n}^{R}$ to be $-\phi_{n}^{R}$, and the prime ( $\left.{ }^{\prime}\right)$ indicates that the sum does not include the $n=0$ term.

The above sequence of phases, where the first few of them are

$$
\begin{aligned}
& \phi_{1}^{R}=x, \\
& \phi_{2}^{R}=2 x-y+f(x), \\
& \phi_{3}^{R}=7 x-4 y+4 f(x)+f\left(\phi_{2}^{R}\right), \\
& \phi_{4}^{R}=26 x-15 y+15 f(x)+4 f\left(\phi_{2}^{R}\right)+f\left(\phi_{3}^{R}\right),
\end{aligned}
$$

is constructed by application of the map (11) on the initial smooth state, $e^{i 2 \pi x}$. Therefore, $|2\rangle,|3\rangle, \cdots$ become increasingly smooth along the unstable manifold and singular along the stable one.

In the same manner we construct the left function by repeated application of the inverse map, namely

$$
\langle L|=\sum_{m=-\infty}^{\infty} A_{m}^{*}\langle m| \quad \text { with }\langle m|=e^{-2 \pi i \phi_{m}^{L}(x, y)},
$$

where the left phases are defined by the recursion relation

$$
\phi_{m}^{L}(x, y)=\phi_{m-1}^{L}(2 x+y, 3 x+2 y+f(2 x+y))
$$

and $\phi_{1}^{L}(x, y)=x$.

There are two simplifying features characterizing $|R\rangle$ and $\langle L|$. First, the operation of the propagator, $U$, on these functions is simple since, by construction,

$$
U|n\rangle=|n+\operatorname{sign}(n)\rangle \text {, and }\langle m| U=\langle m+\operatorname{sign}(m)| .
$$

The second feature is that $\langle L|$ and $|R\rangle$ are orthogonal to a constant function. This way we eliminate the leading Ruelle resonance, $z_{0}=1$, from our problem, since the invariant distribution is constant in phase space. The proof for this orthogonality is straightforward. Let $|0\rangle=\langle 0|=1$ denote the invariant distribution. Then $\langle 0 \mid n\rangle=\left\langle 0\left|U^{n-1}\right| 1\right\rangle=\langle 0 \mid 1\rangle=0$, where we have used the definition of the invariant distribution: $\langle 0| U=\langle 0|$, and that $|1\rangle=e^{2 \pi i x}$ is orthogonal to a constant. Now, from (5) it immediately follows that $\langle 0 \mid R\rangle=0$. Similarly it is straightforward to prove that also $\langle L \mid 0\rangle=0$.

Variation of $F$, given by (湆), (5) and (7), with respect to $A_{m}^{*}$ yields the eigenvalue equation:

$$
\operatorname{Det}[\langle m \mid n+\operatorname{sign}(n)\rangle-z\langle m \mid n\rangle]=0 .
$$

As it stands, this equation is not simpler to solve than the original problem. However, it can be considerably simplified if the matrices $\langle m \mid n\rangle$ and $\langle m \mid n+\operatorname{sign}(n)\rangle$ can be truncated to a small size. As we show below this is indeed the situation. For this purpose, it is instructive to notice few properties of the matrix elements $\langle m \mid n\rangle$. The first one is that

$$
\langle m \mid n\rangle=\langle m-\operatorname{sign}(m) \mid n+\operatorname{sign}(n)\rangle,
$$

where $|m|>1$. This is an immediate consequence of $\langle m \mid n\rangle=\left\langle m \mid U^{-1} U n\right\rangle$, and the relations (8). The second property of the matrix elements comes from the definition of the phases with negative indices, $\phi_{-n}^{L, R}=-\phi_{n}^{L, R}$ :

$$
\langle-m \mid-n\rangle=\langle m \mid n\rangle^{*} \text {, and }\langle m \mid-n\rangle=\langle-m \mid n\rangle^{*} .
$$

From (10) it follows that all matrix elements $\langle \pm m \mid \pm n\rangle$ can be reduced to integrals of the form

$$
\langle \pm 1 \mid \pm l\rangle=\int d x d y e^{2 \pi i\left[\mp x \pm \phi_{l}^{R}(x, y)\right]},
$$

where $l=|m|+|n|-1$. Notice that the above integrals decrease rapidly with $l$. This follows from the nature of the phases $\phi_{l}^{R}(x, y)$. Namely, as $l$ increases, the phases acquire a stronger dependence on $x$ and $y$, see e.g. (6). Thus $\langle m \mid n\rangle$ is small for large $|n|+|m|$, and the significant part of the eigenvalue equation (9) is associated only with a small submatrix.

With this observation, we turn now to calculate the main matrix elements of Eq. (9). First, it is straightforward to see that

$$
\begin{gathered}
\langle 1 \mid 1\rangle=1, \text { and } \\
\langle 1 \mid-1\rangle=\langle 1 \mid \pm 2\rangle=\langle 1 \mid 3\rangle=\langle 2 \mid 2\rangle=0 .
\end{gathered}
$$

Other matrix elements are given by

$$
\begin{aligned}
& \langle 1 \mid-3\rangle=\langle 2 \mid-2\rangle=T_{4}=-\frac{k^{2}}{8}-i \frac{k^{3}}{16}+O\left(k^{4}\right), \\
& \langle 2 \mid-3\rangle=T_{3}^{2}=\frac{k^{4}}{3072}+O\left(k^{5}\right), \\
& \langle 2 \mid 3\rangle=\left|T_{5}\right|^{2}=\frac{k^{6}}{256}+O\left(k^{8}\right),
\end{aligned}
$$

where

$$
T_{\nu}=\int_{0}^{1} d x e^{-2 \pi i[\nu x+f(x)]} .
$$

The above results demonstrate the strong dependence of the matrix elements $\langle m \mid n\rangle$ on $|n|+|m|$, when $k<1$.

\begin{tabular}{|l|l|l|l|l|}
\hline \hline$k$ & $z_{1}$ & $z_{2}$ & $z_{3,4}$ & $z_{5,6}$ \\
\hline 0.1 & 0.035 & -0.037 & $-0.003 \pm 0.050 i$ & $0.002 \pm 0.034 i$ \\
& 0.034 & -0.036 & $-0.001 \pm 0.035 i$ & \\
\hline 0.2 & 0.070 & -0.080 & $-0.009 \pm 0.098 i$ & $0.011 \pm 0.064 i$ \\
& 0.066 & -0.076 & $-0.005 \pm 0.071 i$ & \\
\hline 0.3 & 0.109 & -0.134 & $-0.015 \pm 0.144 i$ & $0.030 \pm 0.088 i$ \\
& 0.094 & -0.12 & $-0.01 \pm 0.11 i$ & \\
\hline 0.4 & 0.153 & -0.196 & $-0.018 \pm 0.188 i$ & $0.066 \pm 0.113 i$ \\
& 0.12 & -0.16 & $-0.02 \pm 0.14 i$ & \\
\hline 0.5 & 0.208 & -0.266 & $-0.023 \pm 0.229 i$ & $0.111 \pm 0.152 i$ \\
& 0.14 & -0.21 & $-0.03 \pm 0.18 i$ & \\
\hline \hline
\end{tabular}

TABLE I. The leading Ruelle resonances of the perturbed cat map at various values of the perturbation parameter, $k$. In each case, the top line is the exact numerical value, while the bottom line is the result of the variational calculation (14). The eigenvalue, $z_{0}=1$, associated with the invariant density is omitted. 

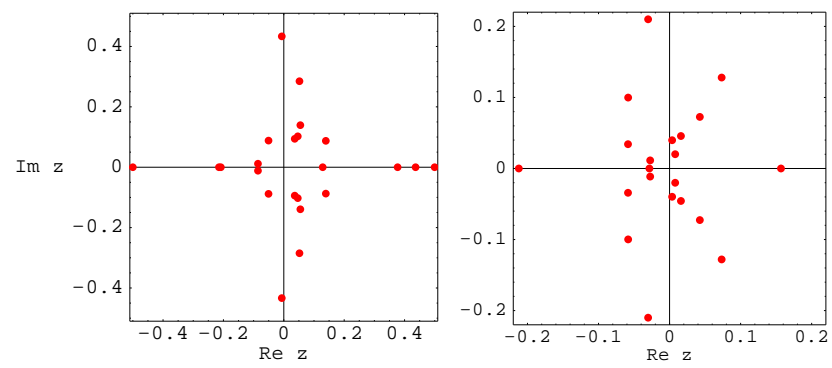

FIG. 2. The configuration, in complex plain, of the Ruelle resonances of two generic chaotic maps. The right panel corresponds the perturbed cat map at $\mathrm{k}=0.5$, and the left panel to the standard map at $K=13$. In both cases the coarse graining parameter is $s=0.005$.

If we truncate the matrices in (9) to $4 \times 4$ matrices, the leading Ruelle resonances are the zeros of the characteristic polynomial:

$$
\operatorname{Det}\left(\begin{array}{cccc}
\left|T_{5}\right|^{2} & 0 & T_{4}^{*} & \left(T_{3}^{2}\right)^{*}-z T_{4}^{*} \\
0 & -z & 0 & T_{4}^{*} \\
T_{4} & 0 & -z & 0 \\
T_{3}^{2}-z T_{4} & T_{4} & 0 & \left|T_{5}\right|^{2}
\end{array}\right)=0 .
$$

These zeros can be calculated exactly, but, having only truncated matrices, it is sensible to evaluate them only to the leading orders in $k$. The results are:

$$
z_{1,2} \simeq \pm \frac{k}{\sqrt{8}}-\frac{k^{2}}{8}, \quad z_{3,4} \simeq \pm i \frac{k}{\sqrt{8}}-\frac{k^{2}}{8} .
$$

In Table I we present the numerical (top line) and the variational results (bottom line) for the Ruelle resonances of the perturbed cat map at various values of the perturbation parameter $k$. The numerical calculation is performed by projecting the equations (11) onto a grid of $90 \times 90$ sites, and calculating the eigenvalues at $s=0$ by extrapolation from the interval $0.001 \leq s \leq 0.005$. It has been checked that the results are independent of the grid, provided the lattice constant is sufficiently small. Comparing the results of Eq. (14) to the exact numerical values, shows that the variational approach provides the order of magnitude and the correct configuration of the leading Ruelle resonances.

To check the generality of this variational approach we turn now to consider the standard mapt as our second example. The standard map is defined by

$$
\begin{aligned}
& x_{n+1}=x_{n}+y_{n} \\
& y_{n+1}=y_{n}+g\left(x_{n+1}\right),
\end{aligned}
$$

where

$$
g(x)=\frac{K}{2 \pi} \sin (2 \pi x)
$$

and $K$ is the stochasticity parameter. The map is integrable when $K=0$, and it becomes increasingly chaotic as $K$ is turned on to a large value. The route into chaos follows the Kolmogorov-Arnold-Moser scenario of breaking of resonant tori. Therefore, the standard map represents a generic mixed system. Yet, for large $K$, the islands of stability are tiny and have negligible influence on correlation functions of sufficiently smooth observables.

Applying the same procedure described above, we obtain that, as before, Eqs. (12) are satisfied, but the other relevant matrix elements are $\langle 1 \mid-3\rangle=\langle 2 \mid-2\rangle=J_{2}(K)$, $\langle 2 \mid-3\rangle=J_{1}^{2}(K)$, and $\langle 2 \mid 3\rangle=J_{3}^{2}(K)$, where $J_{\nu}(K)$ is the Bessel function of integer order. Inserting these results into the truncated eigenvalue equation and calculating its zeros we obtain:

$$
\begin{aligned}
& z_{1,2}=\eta_{+}(K) \pm \sqrt{\eta_{+}^{2}(K)+J_{2}(K)} \\
& z_{3,4}=\eta_{-}(K) \pm \sqrt{\eta_{-}^{2}(K)-J_{2}(K)}
\end{aligned}
$$

where

$$
\eta_{ \pm}(K)=\frac{J_{1}^{2}(K) \pm J_{3}^{2}(K)}{2 J_{2}(K)} .
$$

Thus, to the leading approximation in $1 / K$, the first Ruelle resonances are $17 \pm\left|J_{2}(K)\right|^{1 / 2}$ and $\pm i\left|J_{2}(K)\right|^{1 / 2}$. As evident from Eqs. (16), $z_{1,2}$ diverge whenever $K$ is a zero of $J_{2}(K)$. At these points our variational approach breaks down, but away from them, the results are in agreement with the numerical diagonalization, as demonstrated in Table II.

The intriguing feature of the above results is that the configuration of the leading Ruelle resonances in both examples is similar. The four subleading resonances are located, approximately, at the roots of the equation $z^{4}=\gamma$ where $\gamma$ characterizes the stochasticity of the map. The more stochastic is the map, the smaller is $\gamma$. In particular, for the perturbed cat map $\gamma=k^{4} / 64$ while for the standard map $\gamma=J_{2}^{2}(K)$. It is suggestive that this behavior is generic to a wide class of chaotic maps.

In Fig. 2 we depict the positions, in the complex plain, of the leading 24 resonances of the perturbed cat map and the standard map with a finite coarse graining, $s=0.005$. This figure suggests that the similarity between the classical spectral properties of the map may extend beyond the four subleading resonances. However, at this stage we do not know how to quantify this similarity.

\begin{tabular}{|l|l|l|l|}
\hline \hline$K$ & $z_{1}$ & $z_{2}$ & $z_{3,4}$ \\
\hline 10 & 0.577 & -0.526 & $-0.064 \pm 0.521 i$ \\
& 0.515 & -0.494 & $-0.003 \pm 0.505 i$ \\
\hline 13 & 0.617 & -0.561 & $-0.002 \pm 0.469 i$ \\
& 0.455 & -0.478 & $-0.011 \pm 0.466 i$ \\
\hline \hline
\end{tabular}

TABLE II. The numerical and the variational results for the Ruelle resonances of the standard map at $K=10$ and $K=13$. In each case, the top line is the exact numerical value, while the bottom line is the result of the variational calculation (Eqs. 16). 
It is natural to ask what are the implications of the above results for the spectral statistics of the corresponding quantum maps? To give a partial answer to this question, we consider the form factor which is the Fourier transform of the spectral two-point correlation function. Assuming the map to belong to the orthogonal ensemble, the semiclassical approximation to the form factor is 4

$$
S(n) \simeq 2 n \sum_{\alpha=0}^{\infty} z_{\alpha}^{n}
$$

where $n$ denotes an integer time assumed to be much smaller than the Heisenberg time 18 , and $z_{\alpha}$ are the $\mathrm{Ru}-$ elle resonances of the corresponding classical map. The leading resonance, $z_{0}=1$, is associated with the universal result of RMT, while higher resonances give nonuniversal contributions. As the map becomes more chaotic all $\left|z_{\alpha}\right|$ approach zero except for $z_{0}$, therefore the nonuniversal corrections to RMT become small. Below we show that the configuration of the resonances also plays an important role in suppressing the magnitude of the nonuniversal contributions.

Let us assume that $z_{\alpha} \approx 0$ for $\alpha>4$, and approximate the subleading resonances by $\pm Z$ and $\pm i Z$, where $Z$ is a real positive number smaller than unity. Substituting these $z_{\alpha}$-s in (17) one obtains:

$$
S(n) \approx 2 n+\left\{\begin{array}{cl}
8 n Z^{n} & \text { if } \frac{n}{4} \text { is an integer } \\
0 & \text { otherwise }
\end{array}\right.
$$

From this formula it follows that the nonuniversal corrections to RMT appear only in powers of $Z^{4}$, rather than $Z$. This is a result of cancellations among the contributions of the Ruelle resonances. Thus the magnitude of the nonuniversal contribution to the form factor is dictated both by the absolute values of $z_{\alpha}$-s, as well as by their configuration in the complex plain. It is plausible that other chaotic systems exist where the configuration of resonances lead to an even stronger suppression of nonuniversal contribution, e.g. if the subleading resonances are approximately the roots of $z^{2 \nu}=\gamma$, where $\nu \geq 3$.

To summarize, in this paper we have studied the leading Ruelle resonances of two maps representing typical chaotic behaviors: The perturbed cat map which exhibits hard chaos, and the standard map which is a mixed system. Our analytical and numerical results show that, in both cases, the configuration of the leading Ruelle, in the complex plain, is similar. Numerical studies (e.g. Fig. 2) suggest that the similarities in the classical spectrum of chaotic maps go beyond the properties of the first four subleading resonances. A comprehensive understanding of the classical spectral properties of chaotic systems will open the possibility for understanding the behavior of their quantum counterparts. In particular the weak localization mechanisms associated with quantum interference. In this work we show that the configuration of the Ruelle resonances may result in a suppression of nonuniversal contribution to the form factor. This mechanism of suppression is different from that of diffusive systems in which only the magnitude of the Ruelle resonances is important.

This research was supported by THE ISRAEL SCIENCE FOUNDATION founded by The Israel Academy of Science and Humanities, and by Grant No. 9800065 from the USA-Israel Binational Science Foundation (BSF).

${ }^{1}$ M. C. Gutzwiller, Chaos in Classical and Quantum Mechanics (Springer, N.Y., 1990).

${ }^{2}$ B. L. Altshuler and B. I. Shklovskii, JETP 64, 127 (1986).

${ }^{3}$ B. A. Muzykantskii and D. E. Khmelnitskii, JETP Lett. 62, 76 (1995); A. V. Andreev, O. Agam, B. D. Simons, and B. L. Altshuler, Phys. Rev. Lett. 76, 3947 (1996).

${ }^{4}$ O Agam, B. L. Altshuler, and A. V. Andreev, Phys. Rev. Lett. 75, 4389 (1995).

${ }^{5}$ For a discussion on the relation between the coarse graining parameter and weak localization effects see I. L. Aleiner, and A. I. Larkin, Phys. Rev. E, 55, R1243, (1997).

${ }^{6}$ D. Ruelle, Phys. Rev. Lett. 56, 405 (1986).

${ }^{7}$ B. V. Chirikov, Phys. Repts. 52, 263 (1979).

${ }^{8}$ F. Borgonovi, G. Casati, and B. Li, Phys. Rev. Lett. 77, 4744 (1996).

${ }^{9}$ B. Mandelbrot, The Fractal Geometry in Nature (Freeman, San Francisco, 1982). See also Levy Flights and Related Topics in Physics : Proceedings of the International Workshop Held at Nice, France, 1994 (Lecture Notes in Physic), Eds. M. F. Shlesinger, G. M. Zaslavskii, and U. Frisch (Springer Verlag, 1996).

${ }^{10}$ M. Basilio de Matos and A. M. Ozorio de Almeida, Annals of Physics 237, 46 (1995).

${ }^{11}$ M. L. Mehta, Random Matrices, (Academic Press, New York, 1991)

${ }^{12}$ V. I. Arnold and A. Avez, Ergodic Problems of Classical Mechanics, (Benjamin, New York, 1968).

${ }^{13}$ I. Antoniou, B. Qiao, and Z. Suchanecki, Chaos Solitons \& Fractals 8, 77 (1997).

14 J. P. Keating, Nonlinearity, 4, 277 (1991).

${ }^{15}$ P. Gaspard, G. Nicolis, and A. Provata, Phys. Rev. E 51, 74 (1995).

${ }^{16}$ In this paper we do not discuss the mathematical basis of the variational method. Instead we provide numerical support for its applicability. Further details regarding the mathematical framework of the method can be found in J. L. Schwarzmeier and K. R. Symon, J. Math. Phys. 20, 979 (1979).

${ }^{17}$ A similar result has been obtained recently by M. Khodas and S. Fishman (Phys. Rev. Lett. 83, 2837 (2000)), and by M. Khodas, S. Fishman, and O. Agam (to be published in Phys. Rev. E). Here, by a different method, it has been found that the absolute value of the leading Ruelle resonances is approximately $\left|J_{2}(K)\right|^{1 / 2}$. This method however does not provide the exact location of the resonances in the complex plain neither their degeneracy.

18 The Heisenberg time of quantum maps is the size of the Hilbert space which is inversely proportional to $\hbar$, see J. H. Hannay and M. V. Berry, Physica D, 267 (1980). 